\title{
Knowledge Sharing Barriers in Complex Research and Development Projects: an Exploratory Study on the Perceptions of Project Managers
}

\author{
Vitor Ricardo Santos ${ }^{1,2 *}$, António Lucas Soares ${ }^{1,3}$ and \\ João Álvaro Carvalho ${ }^{2,4}$
}

${ }^{1}$ INESC TEC-Institute for Systems and Computer Engineering of Porto, Porto, Portugal

${ }^{2}$ Department of Information Systems, School of Engineering, University of Minho, Guimarães, Portugal

${ }^{3}$ Department of Informatics Engineering, Faculty of Engineering, University of Porto, Porto, Portugal

${ }^{4}$ Algoritmi Center, School of Engineering, University of Minho, Guimarães, Portugal

\begin{abstract}
Numerous projects are considered complex because of the number of stakeholders, the diversity of skills, and the uncertainty involved, requiring accurate information retrieval and management of the social interactions between different participants leading to efficient knowledge sharing. This paper reports the endings of an empirical study on knowledge sharing barriers and research and development (R\&D) activities that occur in the context of complex project management. The study presents issues, difficulties, and practices acknowledged by project managers related to knowledge sharing and R\&D (focused on activities that involve cooperation and collaboration). Particularly, we point out the following major knowledge sharing barriers: codification process, inadequate information technology, lack of initiative and strategy by the workers, and lack of time and resources. We also explained the following practices and issues regarding the collaborative R\&D activities: information exchange and retrieval, communication barriers, interdependence of knowledge and skills, and different technical terminologies. We intend to contribute to the understanding of the work carried out in the context of complex projects to improve the management practices and the information technology platforms to support them.
\end{abstract}

\section{INTRODUCTION}

This paper reports the findings of an empirical study on knowledge sharing barriers in complex research and development $(\mathrm{R} \& \mathrm{D})$ projects. The study presents issues, difficulties, and practices acknowledged by project managers and participants related to knowledge sharing in $R \& D$ projects, focusing on activities that involve cooperation and collaboration. Particularly, we identify and describe several knowledge sharing barriers in the context of complex $\mathrm{R} \& \mathrm{D}$ projects.
Complex projects are herein viewed as multidisciplinary projects involving $R \& D$ activities (not necessarily in exclusive), carried out by multipartner international teams of different nature (small and medium enterprises, large companies, research centers, etc.) and executed in a geographically distributed environment.

Knowledge sharing barriers in such context include codification, inadequate information technology (IT) to support knowledge sharing, lack of initiative and strategy by the workers, and lack of time and resources. Also, we point out and explain the practices and issues that occur in the collaborative R\&D activities, such as the information exchange and retrieval, communication barriers, the interdependence of knowledge 
and skills between partners and participants, and the difficulties inherent to the use of different technical terminologies. We intend to contribute to the understanding of the work carried out in the context of complex projects so improvement can be brought up to the project work practices and to the IT platforms to support them.

Many projects are considered complex because of the number of participants, the diversity of skills, and the uncertainty involved. Because they demand a high degree of collaboration, complex projects involve social interactions among different participants that enable knowledge sharing. Uncertainty is also considered a key and common aspect of most projects. However, complex projects normally have a high level of unpredicted events, so it becomes difficult to anticipate the impact they will create (Whitty and Maylor, 2009). The growing complexity of project work concerns the increasing number of technical and social relationships and the IT interfaces that must be taken into account by project managers and participants in adapting knowledge from the daily work of a company. Therefore, complexity also increases when different systems grow together; for instance, technical systems merge with administrative business systems, and data are expected to flow between systems (Eriksson et al., 2002).

Project team members often need to apply knowledge they learned in past projects carried out in different contexts. Put in different words, project participants are supposed to apply knowledge that should exist in the organizational memory (Ajmal and Koskinen, 2008). Projects are purposeful activities with a well-defined time limit. So, the organizational memory of a project is something that lasts only for the project duration. The fact that within a project several organizations might be involved, different project members will rely on organizational memories. Knowledge sharing is critical within a project. It affects the development of innovative ideas, the way project members deal with changes, cope with crisis, deal with coordination and complex tasks, define plans, and make decisions (Davidson and Voss, 2002). Nevertheless, knowledge sharing in a project context is "influenced" by each participant's own organizational culture that concerns the norms, values, and procedures associated to the organization and their members (Mian et al., 2008). Nevertheless, project teams face two main problems: first, the teams are usually temporary, so when the project is finished and the team is disbanded, the knowledge associated with the project can be lost; second, it is recommend that communication is improved between team members that work in different projects or locations (Ruuska and Vartiainen, 2005).
Also, Qureshi et al. (2006) argued that distributed project management requires collaboration between organizations and matching right partners to needed capabilities, planning resource requirements, and execution of projects by mobilizing appropriate, dispersed resources to develop or deliver products or services. According to the authors, distributed virtual projects are supporting formal and informal alliances between organizations and groups to meet customer needs. However, the focus is normally put on project control and management and not facilitating on distributed work.

In this study, we focus on complex R\&D projects that aim at creating innovative products, according to some requirements. These projects involve multiorganizational teams, working in a distributed environment and collaborating to make decisions and solve problems.

Knowledge sharing can be viewed as the processes of transferring knowledge from persons, groups, or organizations, which can include relevant information, ideas, and skills (Lee, 2001). In what concerns knowledge sharing in complex projects, there are two knowledge dimensions to be addressed: (1) knowledge about the object of the project, that is, the product to be developed and the technical specifications that leads to a design that meets the requirements, such as pieces, components, parts or assemblies, and/or technologies used; (2) knowledge required to execute the project, that is, work and management structure, schedules, teams and skills involved, relevant scientific and technical knowledge, systems, and interfaces.

The remainder of the paper is organized as follows. First, we present an overview of knowledge sharing and project management involving R\&D activities. Then, the methodology used and theoretical background are addressed. Afterwards, the findings regarding knowledge sharing barriers and $R \& D$ activities are presented. Finally, conclusions and implications for knowledge sharing and project management in complex projects will be discussed.

\section{KNOWLEDGE SHARING AND PROJECT ACTIVITIES}

Knowledge management in the context of a project is the application of principles and processes designed to make relevant knowledge available to the project team. Effective knowledge management facilitates the creation and integration of knowledge, minimizes knowledge losses, and fills knowledge gaps throughout the duration of the project. (Reich, 2007)

Research on multidisciplinary teams and how they interact to overcome barriers and take advantage of their knowledge diversity considers that, to be successful in the global marketplace, organizations 
need to be not only technologically advanced but also use their abilities to create collaboration with partners and share their occupational and cultural knowledge (Ratcheva, 2009). In addition, research on knowledge management showed that IT is the primary mean of preserving coherence, improving information flows, and facilitating contacts between units and groups working in multinational corporations (Lagerström and Andersson, 2003). Empirical studies also concluded that the core of knowledge sharing is social interactions and that IT has just a supportive purpose. Through social interaction, it is possible to establish mutual understanding and trust, allowing the participants to become motivated, committed, and secure in knowledge creating and sharing.

In an organization, knowledge is transferred and shared through the interactions of the employees. Often, this knowledge has been acquired in past experiences. Within multi-organizational projects, knowledge sharing faces difficulties as workers from different organizations are involved. Moreover, when the project is being developed in a geographically dispersed setting, opportunities for interaction among workers belonging to different organizations with immediate feedback and using more than just verbal language are rare.

Following this drive, the work of Goh and Hooper (2009) presents an outline of several barriers to effective knowledge management pointed out by different researchers: lack of trust (Pan and Scarborough, 1999); knowledge regarded as an asset that requires security (Hexmoor et al., 2006); knowledge is leverage over the others (Bartol and Srivastava, 2002); competitive environment within an organization is one of the most relevant factors (Ladd and Ward, 2002; Hansen and Avital, 2005); uncertain or unaware of the value of the information (Goman, 2002); lack of initiative and strategy by the workers in this matter; inadequate information systems and lack of time and resources (Stoddart, 2001); and overpopulated information in the systems and inadequate balance between IT and people (Davenport and Prusak, 1998). Virtual teams face greater challenges in this matter, because they primarily rely on IT to communicate (Powell et al., 2004). The effective building and use of teams is considered vital to the project's success, and the main responsibility of the project manager is to encourage teamwork (Componation et al., 2008). Qureshi et al. (2006) stated the importance of providing communication channels to promote interactions other than those focused on tasks to achieve members' trust, sustained communication, and involvement.

Furthermore, the research of Ratcheva (2009) on multidisciplinary teams and how they interact to overcome barriers and take advantage of their knowledge diversity argues that findings indicate that teams often lack common background knowledge at the beginning of the projects and members are accustomed to different working practices. "Like other projects, transnational projects experience the challenge of getting a diverse group of individuals from different functional areas to work together for a finite period of time to accomplish a specific project objective. Transnational projects, however, face additional challenges: physical distance, cultural diversity, language barriers and technological infrastructure differences" (Adenfelt, 2010).

With regard to leadership, Behrend and Erwee (2009) pointed out that virtual teams present single challenges once they work in geographically distributed environment, usually performing tasks on complex projects that require coordination of inputs and contributions. "A question that arises from this discussion is the metric that would apply to a project to put it into the complex category. This has not currently been established and is required to provide some threshold to the inevitable notion that most projects possess some degree of complexity" (Whitty and Maylor, 2009).

\section{METHODOLOGY AND THEORETICAL BACKGROUND}

The main focus of this study is to describe and explain knowledge sharing barriers that prevent effective collaboration in complex $R \& D$ projects. Our research goal was to study the knowledge sharing behavior and explain the knowledge sharing barriers that occur in this context. Ultimately, we intend to help project managers of complex projects to devise more effective knowledge sharing strategies and to identify key features for IT platforms to support them. The research question addressed is: what are the knowledge sharing barriers in the context of complex R\&D projects?

The results presented here are part of a wider study on the topics of information management, knowledge sharing, and project activities in largescale/complex projects. To accomplish the study purpose, semistructured interviews with subjects from six countries (Portugal, Germany, Spain, UK, Finland, and France) were conducted. This included 24 interviews: 17 face to face, six via video conference on a PC, and one via telephone. This process lasted for 4 months, from November 2009 to February 2010, following a similar approach as seen in the work of Ratcheva (2009). Ochieng and Price (2010) also followed a similar approach for studying organizations in terms of status, sizes, and projects. For that, complex projects participants, researchers, and managers were used to perform the interviews, involving subjects with vast experience in the area from institutions such as research institutes, universities, IT corporations, and industrial associations. The subject's background ranged from mechanical engineering, information systems, multimedia, power systems, industrial management, and construction. 
Open-ended questions that were derived from the research questions and the literature review were used. Before the interview, there was a short conversation with all the participants explaining the context, concepts, and objectives to clarify any misunderstandings. The questions used in the interviews were as follows:

1. In your opinion, what are the main challenges in managing information in projects? Why do you think they happen?

2. Can you please describe how your team usually collects and shares information?

3. Do you use information and/or knowledge management software? What are the main deficiencies that you can identify? How would you improve it?

4. How does your team create and organize the information in the information system? Why it is done in that way?

5. How do you think information management and knowledge sharing could be improved in a project management context?

Interviews were recorded and transcribed, and the interpretation was made using a coding scheme developed according to the literature review. The coding scheme involved a concept map with the previously mentioned there major areas: information management, knowledge sharing, and project management activities. Nevertheless, additional concepts emerged from the coding, as seen in the work of Shachaf (2008), leading to the development of subcategories for the coding scheme.

\section{FINDINGS}

This section addresses the findings regarding knowledge sharing barriers in complex R\&D projects. These categories were used to code what subjects perceived as difficulties, issues, and practices in knowledge sharing and the collaborative $R \& D$ activities related to complex project's work.

NVIVO 8 software (QSR International, Cambridge, MA, USA) was used to support the qualitative analysis of the data gathered during the interviews. This is a commonly used tool for such purpose (Hanisch et al., 2009; Kvale and Brinkmann, 2009; Ochieng and Price, 2010). Each interview is considered a source and each concept of the coding scheme as a tree node. When the identification of a child node was unclear, the concept was coded in the higher node, using a similar approach seen in the work of Ochieng and Price (2010). "In some cases, a particular section would fall into more than one category, but this seemed to indicate the interlinking of themes rather than a fault in coding, for example trust, communication and teamwork" (Ochieng and Price, 2010).
In the following discussion, the findings in each area (knowledge sharing barriers on Table 1; R\&D activities on Table 2) as well as (only some) the key references and the area of work of the participants will be presented, thus providing evidence and promoting the analysis of the knowledge sharing barriers and the $\mathrm{R} \& \mathrm{D}$ activities conducted in the context of complex project management.

\section{Knowledge sharing barriers}

KSB1: codification process

The major barrier pointed to knowledge sharing in complex projects was the codification process, which is related to the following difficulties:

- transferring the knowledge in one's head to paper or digital in an appropriate format (according to the objective or the person's or group's needs);

- incapacity to structure and to share the knowledge in a different format besides the official documents of the project;

- participants consider that knowledge has different levels and that some levels cannot be codified (related to personal point of view);

Table 1 Knowledge sharing barriers results

\begin{tabular}{llcc}
\hline & & Source & Reference \\
\hline KSB1 & $\begin{array}{l}\text { Codification process } \\
\text { KSB2 }\end{array}$ & 6 & 12 \\
& $\begin{array}{l}\text { Inadequate information } \\
\text { technology }\end{array}$ & 3 & 6 \\
KSB3 & $\begin{array}{l}\text { Lack of initiative and } \\
\text { strategy by the workers }\end{array}$ & 3 & 4 \\
KSB4 & Lack of time and resources & 4 & 4 \\
KSB5 & $\begin{array}{l}\text { Learning curve of } \\
\text { information systems }\end{array}$ & 1 & 2 \\
KSB6 & $\begin{array}{l}\text { Competitive environment } \\
\text { KSB7 }\end{array}$ & 1 & 1 \\
LSB8 8 & Unawareness of other & 1 & 1 \\
& people's work & 1 & 1 \\
\hline
\end{tabular}

Table 2 Research and development activities results

\begin{tabular}{|c|c|c|c|}
\hline & & Source & Reference \\
\hline R\&DA1 & $\begin{array}{l}\text { Information exchange } \\
\text { and retrieval }\end{array}$ & 15 & 26 \\
\hline $\mathrm{R} \& \mathrm{DA} 2$ & Communication barriers & 7 & 15 \\
\hline R\&DA3 & $\begin{array}{l}\text { Interdependence of } \\
\text { knowledge and skills }\end{array}$ & 6 & 7 \\
\hline R\&DA4 & $\begin{array}{l}\text { Different technical } \\
\text { terminology }\end{array}$ & 2 & 4 \\
\hline R\&DA5 & $\begin{array}{l}\text { Different organizational } \\
\text { cultures }\end{array}$ & 3 & 3 \\
\hline R\&DA6 & $\begin{array}{l}\text { Different professional } \\
\text { cultures }\end{array}$ & 3 & 3 \\
\hline R\&DA7 & Different work practices & 2 & 3 \\
\hline
\end{tabular}


- documents and initiatives to share knowledge are not appropriate and/or easily understandable, because participants do not know how to conduct a proper approach to knowledge sharing; and

- most of the knowledge is in the key participants' heads (project managers and participants with more experience, and not in regular participants).

... for us it is a problem, how do we structure the knowledge that we acquire in projects in a way that can be retrieved in the future. (x3, Manufacturing Systems Engineering)

It is very difficult to transfer knowledge to paper. (x7, Manufacturing Systems Engineering)

The problem is to use the knowledge in our head and codify it, and sometimes we understand more rapidly a determined matter by talking with someone than reading a document. (x7, Manufacturing Systems Engineering)

I think it starts with the behavior of each one of the participants. Many of them do web searches, gather knowledge, but they are not organized, don't register that information, then when they need that knowledge they have to search for it again. It depends on each one to have training to be more organized, to register that information, that knowledge in a more structured way. (x20, Manufacturing Systems Engineering)

KSB2: inadequate information technology

The second barrier pointed to knowledge sharing was "inadequate IT". This concerns the following aspects:

- tools available to share knowledge are very time consuming and not user friendly (need for automated actions and better interfaces), so people are reluctant to use them;

- different solutions or tools are used (absence of standards or training, requiring a constant learning curve);

- absence of easy communication with other tools and assurance that people really understand the meaning (ambiguity); and

- knowledge sharing systems are mainly process oriented and do not support a more "fuzzy" content.

I think there is still an important lack of real good tools to collect the knowledge. Probably it is not standard and everybody has different means to write and collect the knowledge. But this could be, I do not know any specific tool good to structure and capture the knowledge, and probably the problem is that in each case the tool should be different probably and this is one of the many issues. (x17, Industrial and Power Systems Management, Construction and Software Development)

Probably the main challenge is that, sometimes these tools that are in use now, are tools to share knowledge and this kind of operative knowledge. Sometimes they are not very friendly, and sometimes people are a bit reluctant to use them, because they are very time consuming to get the real information in the real moment. (x17, Industrial and Power Systems Management, Construction and Software Development)

... but in terms of knowledge management, yes it is knowledge but it is not really kind of intelligent in what it does, it is mainly process orientated. Perhaps the main deficiencies, sometimes you are looking for information which might be fuzzier on how it is connected. And, we all got our email boxes and we all got our files systems and we all have our systems like $\operatorname{xxxxx}$ to actually try to find information you want, because sometimes can be one of the most difficult things and, you know, going externally to things like Google. These systems are worse and worse because they find more and more things, things you want but lower and lower down. Getting the information you want is increasingly difficult. (x21, Software Development)

KSB3: lack of initiative and strategy by the workers The third and fourth barriers to knowledge sharing are equally "lack of initiative and strategy by the workers" and "lack of time and resources".

Lack of initiative and strategy by the workers is related to the following aspects:

- "laziness" of the project participants and

- the need to create a culture of collaborative work and knowledge sharing.

One of the main issues is how to structure the knowledge, because it is difficult to structure the knowledge and to put it black and white. It is difficult to represent it and to represent it somewhere with electronic means or not. I think this is the first difficulty and it is caused due to the people, let's say laziness to do it. (x17, Industrial and Power Systems Management, Construction and Software Development)

I think people again, independently how complex a project is, people are always lazy even if they are working in complex environments, humans beings are simple lazy in many cases. So people simply upload the documents, sometimes they add a title and description, for example KnowledgeTree has a 
possibility to define text but nobody types in the text, so that's a problem. (x22, Software Development)

I don't think it's the matter or having an excellent IT infra-structure that will promote knowledge sharing, it's got to be the other way around, people got to be disciplined and motivated to work in the appropriate way, and then an infra-structure that will help and allow the sharing of that information, that knowledge, and not the opposite. (x20, Manufacturing Systems Engineering)

KSB4: lack of time and resources

Lack of time and resources is related to the following aspects:

- absence of proper balance between knowledge codification and time (maximum possible amount and proper format);

- time required to harmonize approaches and common language between partners and participants; and

- existent tools require much time to obtain the approximated or proper results.

... the codification process isn't always objective, so, I write things in a piece of paper, but there is much knowledge that I acquired that is not codified in the paper, because it would take me much time, the issue is to find the adequate format, the faster way to compile the maximum amount of information and knowledge possible. (x3, Manufacturing Systems Engineering)

... a matter of approach. I told you in the beginning that not all the partners are following the same approach by dealing different phases in the project and different topics. Normally there is a need to certain time to harmonize these approaches. I am not sure that any kind of rehearsal of preparations before the project could be better results, there is still a matter of time. I did not see, according to my working life, of course the latest 10 or 15 years of electronic communication being the mostly used that any project the information exchange and knowledge sharing was efficient from the very beginning of the project. There is simply the time needed to harmonize the approaches to find the common language and then it works. Much, of course it is a bit more of understanding of the project topics. Nowadays, you are collecting the teams sometimes in a very short time and simply it is necessary that all of them have a kind common or at least similar understanding of the topics of some complex project. (x12, Industrial and Power Systems Management, Construction and Software Development)
Collaboration in research and development activities

The four major issues related to collaboration in R\&D activities involving large international teams, composed of different types of organizations that work in a geographically distributed environment, are referred to by the participants as information exchange and retrieval, communication barriers, interdependence of knowledge and skills, and different technical terminology.

R\&DA1: information exchange and retrieval

Information exchange and retrieval concerns the following aspects:

- information overload on diverse information systems;

- excessive use of e-mail to exchange information and documents;

- integrating information from different participants and partners; and

- exchanging common (technical) information between participants.

Let's suppose an enterprise that has a production activity, and I am working in the sales department, I got to make a connection with the production department, maybe the person that is working in the sales department is not the same that is working in the production, but there is information that is common and that has to be shared, because I cannot allocate all of the people, there is a task and afterwards there is another. (x3, Manufacturing Systems Engineering)

I would say that the biggest challenge is to integrate all of the information that is gathered during a project, and that is necessary for project management, that's the biggest challenge. Because, we receive information in several ways, information gathered in meetings, information gathered in the teams, information gathered from clients, in different formats, it can be in paper, email, etc.. The biggest difficulty, is without doubt in integrating information and having a global overview, and also updated the information, I would say that is the biggest challenge. (x9, Information and Computer Graphic Systems)

The number one method to share information is email, which of course is not the best way to do it still is the one that everybody uses and everybody is using for all other purposes, so I would say where in number one is email lists with all the people on it. In big projects you have multiple email lists that are used to separate and share information. (x22, Software Development) 
The main challenge is to pass the requirements through the chain, because maybe there is a big company and there is a costumer, so they are a global company they have costumers all over the world and those costumers give small piece of information, and they should collect this information. There is a huge amount, lot of costumers, huge amount, what is important, what is less important, so, how they could filter this vast information from the information, let's call it must have information or knowledge. (x24, Software Development)

I receive tons of emails during the day, during the month I am exchanging thousands of emails. So, who is my key partner? I should give different priorities with people I am collaborating, and maybe I could exchange. (x24, Software Development)

\section{R\&DA2: communication barriers}

The second major aspect is "communication barriers" that regard the following:

- difficulties in establishing a common technical language understandable by all participants;

- presenting information in an appropriate timing and structure;

- personal backgrounds, time zones, national cultures, and technical contexts (leading to misunderstandings and conflicts);

- difficulties in communicating with and managing expectations and requirements of the clients; and

- use of miscellaneous technologies (e-mail, videoconference, and portals) to try to deal with challenges (however to solve problems, according to the participants, it is better to have personal interactions such as meetings or conversations).

One of the challenges is having the information in a format that is understandable by everybody, so, creating a common communication language that is one of the major challenges. There is a great need to produce information, each element of a determined group has always the need to translate the information using the internal codification that is inherent to the organizational culture where he belongs, translated to a format different that is understandable by everybody. There is an effort to produce information in a common language, and because that language does not exists, many times that process is not efficient. (x11, Mechanical Engineering and Industrial Management)

In European projects this is something that we see a lot, time zone barrier, language, certain details, because the person on the other side, with whom we are communicating not always understands the language that we are using. And, we have lots of examples, for instance right now we are working in a European project were that happens. We were requested to provide information about the use of resources of $\mathrm{xxxxx}$, and I wrote a letter, explained everything, we followed the procedures, and the person on the other side wants financial information about xxxxx. They still didn't understand that there is an agreement of the use of human resources on xxxxx from xxxxx. (x14, Power Systems)

... one of the difficulties we have, when we are distributing technical tasks, we ask partners to give contributions, and what we see is that people send contributions that sometimes are not adequate to the objectives they propose. And, most of the times its better that the person who is managing, because has an overview of the objectives adapts the contributions. Because, it has a more global overview of what to ask from the partners, or things start to be sent back and forward, and we have to explain that things are not like that, what we intend to do is this, and then he sends things back again, and starts the ping-pong that usually exceeds the deadlines. (x14, Power Systems)

When you have a project going on with some objective, you have to manage quite well the expectations of the client, because many times you are talking about a determined functionality of the software and he is understanding one completely different, totally different. For instance, you can be, now using examples, on a project that we have undergoing in planning area, we are concerned in solving a planning problem, and the client thinks that the algorithm that we are programming will solve the problem of the enterprise. And we realized this is not true, it's an organizational problem, it is not the software that is going to solve the problem, and it is a problem of internal organization, it has to be solved first and then the application will help in decision making. So, it starts there, what the client understands, what is his problem and then we try to understand the problem and solve it... (x20, Manufacturing Systems Engineering)

If you are developing that sort of project internationally then of course you get very understandingly things happen just in terms of communication difficulties. We might like to think we could all work offline, but in time we use Skype, and email, and teleconference and all this kind of stuff, but the reality there is nothing like people seating down together in the same room, and sitting down in that room doing the work day, but also sitting down with that person in the bar afterwards to talk problems through into brainstorm same ideas. (x21, Software Development) 
One of the big things we find in multi-partner, multinational projects is a lot of people do rely on email now and of course what we find on many instances, people say things in email which might sound different in email or wouldn't say to you verbally. So things often kind get escalated for no reason and sometimes even become rude, it is not because of the individuals it is kind of the individual use of the emails. (x21, Software Development)

R\&DA3: interdependence of knowledge and skills The third most focused aspect was interdependence of knowledge and skills. This matter concerns the need for working with different participant's knowledge and skills, because complex projects are executed by multidisciplinary teams. Obviously, this raises practical issues because, as subjects stated, each professional domain has its own technical and professional terminologies and way of working, and because complex projects require a wide variety of specialists, the collaboration of a large variety of knowledge domains is necessary. Another issue to take into consideration is that organizations, principally organizations that deal with sensitive information or knowledge (for instance, $\mathrm{R} \& \mathrm{D}$ projects using cutting edge technology), tend to work in a protective manner restricting the exchange of knowledge and skills. This matter is negatively affected by the nature of the work, once it is executed in a distributed environment, leading to effort duplication or some areas not being approached at all as one of the participants said.

Most of the times projects are multi-disciplinary, what I mean with this, I can be working in several domains, despite our flexibility there are specializations. I am the most generic element, so I have to know a little from everything, but on my team there are persons specialized in certain domains. When a project has a certain dimension, what is usual, it is necessary more than one specialist, but there is information that is necessary to exchange. (x3, Manufacturing Systems Engineering)

Industry might know little bit about it, but for academics it is all very simple in the research state, but in the development stage academics tend to know less and commercial people know more, and you are getting different types of persons involved and you are also getting these parties from different countries and culturally this could be quite difficult. (x21, Software Development)

R\&DA4: different technical terminology

The fourth aspect stated by the participants is difficulties as a result of different technical terminology.
This aspect if culture is important or not, evidently yes it is. And, just to confirm that, when we are talking about internal culture, it has much to do with the technical and scientific culture that people bring to the teams. Specifically issues related with reference models, lexis and sometimes even semantics, for instance, if I have an economist, someone with a degree in economy, he has a very different language that an engineer, how will he adapt and understand these issues. Even inside engineering, each school, each domain and each specialization has its own. (x3, Manufacturing Systems Engineering)

There is an interesting aspect that projects, a project gathers multi-disciplinary contributions and multi-disciplinary contributions have their own code, their own culture, their own language... (x11, Mechanical Engineering and Industrial Management)

... what happens many times is that information has different formats, has different rules, uses different applications to be codified, uses, we use different technical terms that are professional technical dialects, so it is not easy to automate these processes. (x11, Mechanical Engineering and Industrial Management)

There is much work to be done here, because this codification oriented to certain attributes, first of all the definition of attributes, isn't it? Attributes, it is necessary to know what to use and that constitutes by itself a new language. So, what it used is the dialect of the technical area that allows schematics in a determined way, but evidently it would be interesting if we could evolve to a common language, and that allowed to work inside the organization but also interacting with the exterior, but that is not possible.. . (x11, Mechanical Engineering and Industrial Management)

\section{CONCLUSIONS AND IMPLICATIONS}

\section{Knowledge sharing barriers}

The results present a noticeable high number of references to the major knowledge sharing barrier: codification process. Although the subjects are aware of the importance of knowledge sharing, they are concerned on how to structure the knowledge they acquire during projects in a way they can use it in the future. Some even consider that it is possible to share it in the documents they created during the project, but they agree that it is complicated to do because it takes much time to codify knowledge in an appropriate format. For them, it still is a questionable matter to know how much knowledge can be codified, because as said earlier, 
most matters are not written. Some participants even consider that this codification issue is unsolvable, because according to them, in complex projects environment, they understand more rapidly a determined matter/technical solution by talking to someone than reading a document.

The subjects consider that complex projects have two important aspects that must be addressed: project-specific content and the necessary dynamic of working in complex projects environment (which also requires learning). This is a kind of knowledge that is acquired when interacting with different participants and solving problems and is gathered during projects and improved with experience. It evolves and improves the efficiency of the next projects: the way teams work in projects; however, this knowledge is usually lost. The participants consider that is not easy to codify this kind of knowledge, because it is only maintained individually by members, and the only persons that usually carry that knowledge are the key persons that have core knowledge as a result of their experience.

Another level of knowledge that the subjects consider to be lost is the way people gather information to perform their work-their techniques to work better and faster. They believe that this kind of knowledge cannot be registered anywhere, because it is much related to personal experiences. However, it is argued that with proper training, participants can be more organized, and the way people register knowledge can be improved in a more structured and easily retrievable way.

The second major barrier to knowledge sharing pointed out by the subjects was inadequate IT, which is related to the major knowledge sharing barrier, because the participants argued that the codification process is also hampered by the absence of appropriate information systems. In general, they think that there is still an important lack of real good tools to collect knowledge, and the way it is performed is not standard. Also, there are different ways of writing and collecting knowledge, and existent tools work differently and are not user friendly. These aspects make people reluctant to use them, because it becomes time consuming to insert data and, as told before, in complex projects environment, time is considered very scarce. Another level of difficulty is that people consider knowledge sharing as a fuzzy domain or concept and that, when sharing knowledge, it is not possible to be sure if they are dealing with the right knowledge-really sending the appropriate message.

The participants consider that existent knowledge sharing tools do not provide easy and friendly integration with other systems, and because complex projects knowledge is fuzzier and is scattered in e-mails, web portals, and administrative and technical information systems, there is still much work to be carried out. The inadequacy of the information systems can be worsen by the lack of time, because knowledge sharing information systems require much workload, for instance, people have to $\log$ in, find and navigate to the right folder, upload or create the document, add information, and still inform other participants that a document has been created or uploaded. So, what happens in reality is that people use knowledge management systems for some weeks and then switch back to e-mail. The subjects consider that the main reason for that is it requires too much time. They are aware that it only requires a few extra seconds, but for the participants, it is still much faster to open an e-mail and attach a file.

The subjects pointed another barrier as the lack of initiative and strategy by the workers and that knowledge sharing difficulties are caused by people's "laziness" despite how complex an environment is. And, it is necessary to take measures for creating a culture of people working together and sharing knowledge. According to what has been expressed, it is not only the matter of having an excellent IT infrastructure that will promote knowledge sharing but also people must have training and be motivated to work in an appropriate way. Hanisch et al. (2009) support this issue when they stated that organizational culture seems to be an important factor of successful project knowledge management. It is considered that IT systems and methodologies alone for supporting knowledge gained in projects are useless if the employees resist using them. Although, the combination of high quality IT system and a systematic approach that fits the needs of the project and the organizational structures is considered to support management of project knowledge.

Lack of time and resources was pointed as another knowledge sharing barrier. Because of complex projects multipartner environment, there must be a harmonization of approaches, because in the beginning of the project, not all partners are following the same approach to different phases and topics of the project. However, currently, project managers have to deploy teams in short time and simply it is necessary that all of them have a similar understanding of the topics of a complex project. The work of Ratcheva (2009) on multidisciplinary teams and how they interact to overcome barriers and take advantage of their knowledge diversity confirms this issue, because she argues that teams often lack common background knowledge at the beginning of the projects and members are accustomed to different working practices. In addition, there is the issue of the competitive environment where there is some reluctance in sharing knowledge. People understand knowledge as power, and because teams that work in geographically distributed environment face greater difficulties in building trust, it is important to have regular meetings to diminish this issue. 
This also can lead to unawareness of other people's work where reinventing the wheel is very common, because one may be on the same team but not on the other. So, if parameters, tests, and experiments were already carried out and the team does not know about it, then they will perform the task again. As a result, the subjects consider that is important that knowledge should not be restricted to one particular project but should be accessible for the next projects, therefore having a crucial impact in the effectiveness of one or other projects.

These aspects are partially explained in the work of Lin (2008) where he presented the idea that the lower the formalization of an organizational structure, the greater the knowledge sharing among units of an organization, whereas the higher the complexity of an organizational structure, the lower the knowledge sharing among units. Higher trust and commitment are also important to foster knowledge sharing among individuals for successful knowledge sharing activities.

The results presented are consistent with the conclusions of van den Hooff and Huysman (2009) where it is stated that management could influence knowledge sharing by promoting little formal barriers in interaction between different parts of the organization and establishing and maintaining an IT infrastructure that efficiently and effectively helped organizational members to learn what relevant knowledge is. However, the authors are aware that the direct influence of management measures may be limited, because it is primarily with social group interactions that knowledge sharing is stimulated.

The conclusions are also confirmed by the work of Reed and Knight (2010) where it is considered that knowledge sharing on colocated projects takes place informally, through water cooler or over-the-cubicle remarks. The authors further believe that sharing undocumented knowledge and face-to-face exchange of information can be difficult to accomplish in a virtual environment. The solution they provided is consistent with the results presented earlier where it is stated that the documentation should be emphasized, which can then be exchanged electronically.

\section{Research and development activities}

Research and development activities, specifically the ones that involve a high degree of cooperation and collaboration through information exchange and retrieval, according to the participants are carried out essentially using e-mail, where they considered that too much time is spent exchanging e-mails and trying to get feedback. This aspect is confirmed in the work of Shachaf (2008) where it is considered that multination corporations use e-mail for intercultural communication with teleconferencing coming second. The participants stated that complex projects require accurate connection between partners because information is common and needs to be shared. Hong Joo et al. (2006) summarized the current issues and state of the problems where they expressed that although efficient management of knowledge and collaboration in engineering changes is crucial for the success of a new product development, systems focus mainly on storing documents or simply automating the approval process, while knowledge that is generated from collaboration and decision-making processes may not be captured and managed easily.

However, most participants consider that, in complex projects, there is the problem of the information overload, where too much information is sent by e-mail. So, they consider that it is necessary to send only the strictly important information; otherwise, a large volume of e-mail will be generated. The exchange and retrieval of information can be seen in structured documents: produced by someone, e-mailed to the team, the team responds, and the document moves back and forth. Following this drive, the biggest challenge is to integrate the information that is gathered during a project, and this is necessary for project management. Information is received and exchanged in several ways-in meetings, by teams, from clients, in different formats-so it is necessary to integrate information and provide a global overview.

Information exchange and retrieval is also related to the distribution of tasks, where partners are asked to contribute requiring from project manager an overview of the objectives, selection, and adjustment of contributions. If the project manager is not involved actively, information starts to be sent back and forth, ending in the earlier called "ping-pong" that exceeds the deadlines. The information exchanged can be administrative or technical, for instance, parameters of cables, regulators, and generators. As said earlier, the major communication tool to share information is e-mail. As a result, multiple e-mail lists are created and used to separate and share information. This raises practical issues, because mailing lists are created for different groups and some subjects participate in all the groups. So, when a problem is raised that is related to all the partners, everybody responds and the participants see one answer after the other when the solution was already given. According to them, this increases the information overload even more.

These issues lead to the vast amount of e-mails received during the day, instead of giving different priorities to the key partners and subjects. Communication barriers present several challenges to address, for instance, having the information in a format that is understandable by everybody, so creating a common communication language during a project is one of the issues. As pointed out by Reed and Knight (2010) who argued that communication is essential for efficient coordination and that participants must be working "on the same page", 
participants have to convert the information using the internal codification language that is inherent to the organizational culture to a format that is understandable by everybody. This is due to the participant's different backgrounds, contexts, and cultures. Most of the time, despite the availability of information, it is interpreted differently from what was intended by the creator.

Because of the different cultures and languages and although it is possible to use several technologies such Skype, e-mail, and teleconferences to solve issues, they consider that, in reality, there is nothing like participants having a meeting or talk in a bar after work to discuss problems in a brainstorming session. Also, the subjects pointed that people express matters on e-mail, which might sound different, or would not say it verbally and sometimes matters get escalated for no reason and even can become rude. This is not due to an individual's deliberated misuse of e-mail but to the individual use of e-mails.

A characteristic of complex projects is the multidisciplinarity and the necessity of interdependence of knowledge and skills. Participants have to work in several domains despite individual specializations, and because projects have a big dimension, it is necessary to have several specialists. But there is information that is common and has to be exchanged. One way to address this is to have complex project participants working in open spaces, where they are naturally encouraged to share information between projects, not only inside a determined project but also in different projects. In this aspect, communication becomes easier. It is possible to clear doubts and find someone with an appropriate know-how for a project.

Although there is the need for interdependence of knowledge and skills, collaboration is complicated. It is not efficient because of the habit of institutions performing their work independently from others, leading to effort duplication, or there are aspects that are worked in different ways or some areas not being addressed. These issues regard the fact that complex projects gather multidisciplinary contributions, and these have their own code, culture, and language. These contributions can include the academics in the research state, professional developers, and commercial people, and project managers have to integrate all these types of participants. Because these parties are from different countries, culturally this could be quite difficult.

The subjects consider that the different technical terminologies present challenges, because they are related to the technical and scientific culture that participants bring to the teams, specifically issues related to reference models, lexis, and even semantics. A person with a degree in economy working in a project has a different language from an engineer. Even in engineering, each school, each domain, and each specialization have their own language.
In addition to this discussion, information has different formats, different rules, and uses different applications to be codified, and participants use different technical terms that are professional dialects, thus hampering the automation of processes. So, in the future, it should be advisable to move from the dialect of the technical area and eventually evolve to a common language that would allow not only working inside the organization or project but also interacting with the exterior. Subjects also have to deal with different company's organizational cultures or policies that forbid communication tools such as Skype and other technical restrictions, presenting a challenge when interconnecting with different companies.

The participants consider that the issue of different work practices can be seen, for instance, in deliverables where different approaches are taken to the description of the work that is carried out. Each organization describes it in its own way, and because it has to be created as a unique document and there is not a single structure, it is problematical to adjust contributions and make it coherent. Also, individually people work and have different routines, some like to work early in the day and some at the end of the day, and tasks are addressed in different ways. This issue is associated with the difficulties with different members, groups, or entities and the sheer number and type of partners, because in complex projects, small and large companies, universities, and research institutes are integrated, and it is necessary to combine them together.

Ochieng and Price (2010) argued that the absence of face-to-face communication can lead to misunderstandings, and Shachaf (2008) considered that collaborative group systems should incorporate possibilities of using multiple media channels. The work performed in geographically distributed environment, according to the subjects, requires a large volume of communication, leading to several problems. It should take into consideration that technology based on the Internet, e-mail for instance, usually raises these misunderstandings. However, it is advisable to adopt communication tools such as videoconference where persons could be seen. The results indicate that seeing the other participants helps, unless complicated matters are to be addressed. In that case, a face-to-face approach is required. But for the normal task, this is advisable because it avoids costs and time.

\section{REFERENCES}

Adenfelt M. 2010. Exploring the performance of transnational projects: shared knowledge, coordination and communication. International Journal of Project Management 28(6): 529-538. DOI: 10.1016/j.ijproman.2009.10.004. Ajmal MM, Koskinen KU. 2008. Knowledge transfer in project-based organizations: an organizational culture perspective. Project Management Journal 39(1): 7-15. 
Bartol KM, Srivastava A. 2002. Encouraging knowledge sharing: the role of organizational reward systems. Journal of Leadership and Organizational Studies 9(1): 64-76.

Behrend FD, Erwee R. 2009. Mapping knowledge flows in virtual teams with SNA. Journal of Knowledge Management.

Componation PJ, Youngblood AD, Utley DR, Farrington PA. 2008. A preliminary assessment of the relationships between project success, system engineering, and team organization. Engineering Management Journal 20(4): 40-46.

Davenport TH, Prusak L. 1998. Working Knowledge: How Organizations Manage What They Know. Harvard Business School: Boston.

Davidson C, Voss P. 2002. Knowledge Management: An Introduction to Create Competitive Advantage from Intellectual Capital. Tandem Press: Auckland.

Eriksson M, Lillieskold J, Jonsson N, Novosel D. 2002. How to manage complex, multinational R\&D projects successfully. Engineering Management Journal 14(2): 53.

Goh CHT, Hooper V. 2009. Knowledge and information sharing in a closed information environment. Journal of Knowledge Management.

Goman CK. 2002. Five reasons people don't tell what they know. Retrieved from: http://www.providersedge. com/docs/km_articles/Five_Reasons_People_Dont_Tell_ What_They_Know.pdf

Hanisch B, Wald A, Lindner F, Mueller A. 2009. Knowledge management in project environments. Journal of Knowledge Management 13: 148-160.

Hansen S, Avital M. 2005. Contributing your 'wisdom' or showing your cards? A quantitative inquiry of knowledge sharing behaviour. Paper presented at the Proceedings of the 11th Americas Conference on Information Systems.

Hexmoor H, Wilson S, Bhattaram S. 2006. A theoretical interorganizational trust-based security model. Knowledge Engineering Review 21(2): 127-161.

Hong Joo L, Sung Joo P, Hyung Jun A, Jong Woo K. 2006. Capturing and reusing knowledge in engineering change management: a case of automobile development. Information Systems Frontiers 8: 375-394.

van den Hooff B, Huysman M. 2009. Managing knowledge sharing: emergent and engineering approaches. Information Management 46: 1-8.

Kvale S, Brinkmann S. 2009. Interviews Learning the Craft of Qualitative Research Interviewing, 2nd edn. SAGE: Los Angeles [etc.].

Ladd A, Ward MA. 2002. An investigation of environment factors influencing knowledge transfer. Journal of Knowledge Management Practice 10(1).

Lagerström K, Andersson M. 2003. Creating and sharing knowledge within a transnational team - the development of a global business system. Journal of World Business 38(2): 84-95. DOI: 10.1016/S1090-9516(03)00003-8.

Lee JN. 2001. The impact of knowledge sharing, organizational capability and partnership quality on IS outsourcing success. Information Management 38(5): 323-335.

Lin WB. 2008. The effect of knowledge sharing model. Expert Systems with Applications 34(2): 1508-1521.

Mian A, Takala J, Kekale T. 2008. Role of organizational culture for knowledge sharing in projects. 2008 Portland International Conference on Management of Engineering \& Technology 1-5: 962-968.

Ochieng EG, Price ADF. 2010. Managing cross-cultural communication in multicultural construction project teams: the case of Kenya and UK. International Journal of Project Management 28(5): 449-460. DOI: 10.1016/ j.ijproman.2009.08.001.

Pan SL, Scarborough H. 1999. Knowledge management in practice: an exploratory case study. Technology Analysis \& Strategic Management 11(3): 359.

Powell A, Piccoli G, Ives B. 2004. Virtual teams: a review of current literature and directions for future research. SIGMIS Database 35(1): 6-36.

Qureshi S, Liu M, Vogel D. 2006. The effects of electronic collaboration in distributed project management. Group Decision and Negotiation 15(1): 55-75.

Ratcheva V. 2009. Integrating diverse knowledge through boundary spanning processes - the case of multidisciplinary project teams. International Journal of Project Management 27(3): 206-215.

Reed AH, Knight LV. 2010. Effect of a virtual project team environment on communication-related project risk. International Journal of Project Management 28(5): 422-427. DOI: 10.1016/j.ijproman.2009.08.002.

Reich BH. 2007. Managing knowledge and learning in IT projects: a conceptual framework and guidelines for practice. Project Management Journal 38(2).

Ruuska I, Vartiainen M. 2005. Characteristics of knowledge sharing communities in project organizations. International Journal of Project Management (5 SPEC. ISS.) 23: 374-379.

Shachaf P. 2008. Cultural diversity and information and communication technology impacts on global virtual teams: an exploratory study. Information \& Management 2: $131-142$.

Stoddart L. 2001. Managing intranets to encourage knowledge sharing: opportunities and constraints. Online Information Review 1: 19-29.

Whitty SJ, Maylor H. 2009. And then came Complex Project Management (revised). International Journal of Project Management 27(3): 304-310. 